\title{
Ground-based synthetic aperture radar interferometry for deformation monitoring: a case study at Geheyan Dam, China
}

\author{
Qihuan Huang, ${ }^{a}$ Guido Luzi, ${ }^{b} *$ Oriol Monserrat, ${ }^{b}$ and Michele Crosetto ${ }^{b}$ \\ ${ }^{a}$ Hohai University, School of Earth Sciences and Engineering, Jiangning District, Nanjing, China \\ ${ }^{\mathrm{b}}$ Centre Tecnològic de Telecomunicacions de Catalunya (CTTC/CERCA), Geomatics Division, \\ Castelldefels, Spain
}

\begin{abstract}
Full coverage and continuous deformation information retrieval are key aspects for dam health diagnosis. Ground-based synthetic aperture radar (GB-SAR) interferometry is used for the remote monitoring of the Geheyan Dam, China. Although the monitoring of a dam with ground-based interferometry is not an innovation, specific issues have been found out in the case study discussed due to the large dimension of the monitored structure. More than 400 images were used for interferogram generation. Radar signals reflected from the dam were carefully analyzed: a sort of tunneling effect caused by multireflection is observed, and deformations caused by water level and temperature variations were detected during a six-day monitoring campaign. Radar monitoring results were compared to the data recorded by plummets installed in the dam. The agreement between the displacements retrieved from interferometric data and the plummets demonstrates the capability of GB-SAR for deformation monitoring, with the advantage of large area coverage.
\end{abstract}

Keywords: ground-based radar; interferometry; dam; deformation.

\section{Introduction}

To monitor the health-state of a dam, the detection and estimate of small deformations are crucial. Pointwise deformation measurement techniques, such as the Global Positioning System, leveling, and plummet, are usually employed for data collection. These sparsely distributed points are used to describe the movement of the entire dam, and deformation details might be overlooked due to the lack of points, especially when the dimension of the monitored structure is large. Furthermore, these field data collection activities are labor intensive and, in some situations, even unsafe. Terrestrial laser scanning allows capturing dense 3-D point clouds with a high degree of automation, but the accuracy and effectiveness of this technique strongly decreases with sensor to target distance and with adverse atmospheric conditions.

Ground-based synthetic aperture radar (GB-SAR) for deformation monitoring has consolidated in the last decade as a tool able to provide displacement maps with high spatial and temporal resolution and accuracy in different situations. ${ }^{1,2}$ It is a radar-based terrestrial remote sensing imaging system that exploits the interferometric capability of centimeter wavelength microwaves to obtain submillimeter to millimeter deformation estimates. ${ }^{3}$ Although not widespread as spaceborne SAR interferometry, GB-SAR interferometry has proven to be a reliable remote sensing tool.

The GB-SAR technique has been used to monitor a wide range of deformation phenomena, such as volcanoes, ${ }^{4}$ landslides,,${ }^{5,6}$ and glaciers, ${ }^{7}$ and open-pit mine monitoring, ${ }^{8}$ and also for topographic mapping. ${ }^{9,10}$ The use of GB-SAR for dam deformation monitoring dates back to $1999^{11}$ and was further demonstrated by Alba at al. ${ }^{12}$ The dams' front profiles monitored in both cases are relatively small and flat, with easily interpreted backscattering properties.

*Address all correspondence to: Guido Luzi, E-mail: gluzi@cttc.cat 
In this work, a dam with a large complex front profile is monitored. The dam here monitored has been already surveyed in a previous study, ${ }^{13}$ but with a different geometry, from a shorter range and for $8 \mathrm{~h}$ only. Xing et al. ${ }^{13}$ focused on the environmental effects affecting the radar acquisition, even though the limited range and short temporal duration of the monitoring reduce the influence of atmospheric propagation with respect to the case analyzed in this paper. In this paper, different behaviors of radar signals reflected from different parts of the structure are analyzed, aimed at estimating deformations during a longer lapse, and from a large distance. The main results of the monitoring in this paper consist of deformation time series covering more than $120 \mathrm{~h}$, where the influence of daily atmospheric fluctuations is detected and corrected. The outcomes are compared with daily plummet measurements to assess the reliability of the proposed approach. The relationship between the behavior of deformation along time and the reservoir water level and temperature variation occurred during the period surveyed are analyzed.

\section{GB-SAR Deformation Monitoring}

\subsection{GB-SAR System}

The GB-SAR is a monitoring system mainly composed of the following four separate modules: (1) a radar sensor able to transmit and receive microwave signals, (2) a mechanical scanning rail, $2-\mathrm{m}$ long in the case at hand, along which the sensor moves providing synthetic aperture imaging, ${ }^{3}$ (3) a laptop for data acquisition and processing, and (4) a power supply module that allows continuous measurements. Many types of GB-SAR systems have been developed in the last decades. A review of the GB-SAR system used in this work can be found in Ref. 14. All the applications of this technique need to carefully deal with the atmospheric artifacts, which are the main source of error in GB-SAR interferometry. ${ }^{15}$ Atmospheric phase screen (APS) studies and related solutions have been proposed by different authors. In Sec. 3, where the data processing chain and strategy are described, this issue will be addressed.

Figure 1 shows a general view of the IBIS-L GB-SAR system marketed by IDS Ingegneria dei Sistemi SpA, which was used for data collection in the Geheyan Dam area.

\subsection{GB-SAR Interferometry for Deformation Monitoring}

Ground-based radar interferometry is based on the same principles as satellite interferometry. SAR interferometry exploits the phase difference between two temporally separated SAR observations; the observed phase of each pixel of an SAR image contains information about its distance from the GB-SAR sensor. If decorrelation is negligible, an interferogram can be generated using two SAR acquisitions by computing the phase difference between these two images. The differential phase obtained is, in the simplest case, proportional to the variation of the line-ofsight (LOS) distance. With respect to satellite SAR interferometry, where each image is acquired from a different position (due to the orbit constraints) introducing a spatial baseline,${ }^{16}$ in the case of ground-based observations, the radar is maintained in the same position, i.e., with zero baseline. Assuming that the dielectric characteristic of the pixels of the SAR image remain unchanged between two SAR acquisitions, the interferogram generated reveals the displacement

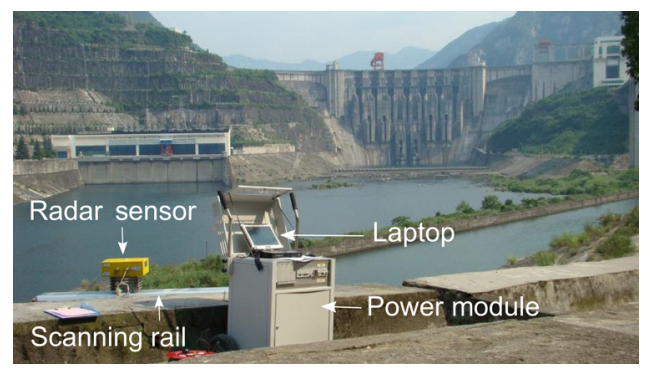

Fig. 1 IBIS-L system installed at Geheyan Dam area. 
of the observed surface occurring in the observed period. The displacement associated with a pixel can be recovered using the following simple equation:

$$
d_{x}=\frac{\lambda}{4 \pi} \Delta \varphi_{x},
$$

where $\lambda$ is the wavelength of the radar sensor and $\Delta \varphi_{x}$ is the phase difference (interferometric phase) of pixel $x$. Interferograms can be obtained following two different approaches: by comparing pairs of images acquired at different times separated by the same interval or taking an image as a reference and one acquired at a different increasing time. In the first case used in this data processing, wrapping and decorrelation are reduced. ${ }^{11}$

In reality, decorrelation caused by geometrical, instrumental, and temporal causes can affect the estimated displacement. Geometrical decorrelation is minimized by installing the GB-SAR system in a stable position on a concrete base. The performances of the radar sensor and the stability of backscattering characteristics of the monitored surface are responsible for maintaining a negligible decorrelation along the whole image acquisition duration.

\subsection{Measurement Setup}

The Geheyan Dam is located in the Qingjiang River, a tributary of the Yangtze River, surrounded by high mountains on both sides. The climate of the area is subtropical continental, with cold winters and hot and humid summers. The Geheyan Dam is well-known for its singular design: a gravity dam on the lower part and an arch dam on the upper part. The crest elevation is $206 \mathrm{~m}$, the maximum dam elevation from bottom to top is $151 \mathrm{~m}$, and the arc length is $648 \mathrm{~m}$. The overflow section is located at the middle of the dam and has seven surface orifices (the highest at $181.8 \mathrm{~m}$ ), four middle orifices (the highest at $134 \mathrm{~m}$ ), and two deep orifices (the highest at $95 \mathrm{~m}$ ) mounted with radial gates. The orifices dimensions (width $\times$ height) are $12.0 \times 18.2 \mathrm{~m}, 4.5 \times 6.5 \mathrm{~m}$, and $4.5 \times 6.5 \mathrm{~m}$, respectively. The powerhouse is located on the right bank terrace of the river and a vertical ship lift stands on the left bank.

The IBIS-L system was installed on a concrete stable platform on the left bank terrace at a distance of $\sim 1300 \mathrm{~m}$ (Figs. 1 and 2). The main parameters of the system are listed in Table 1. 1330 GB-SAR images were collected between July 27 and August 2, 2013, totaling an acquisition period of 5 days, $14 \mathrm{~h}$, and $48 \mathrm{~min}$. These images were acquired in an almost continuous mode, a time interval between image acquisitions of about 5 min, with the exception of some breaks caused by power supply interruptions. Figure 3(a) shows the mean amplitude image with the dam area highlighted by a white rectangle. The pixel selection was based on the amplitude dispersion index $\left(D_{A}\right)^{17}$

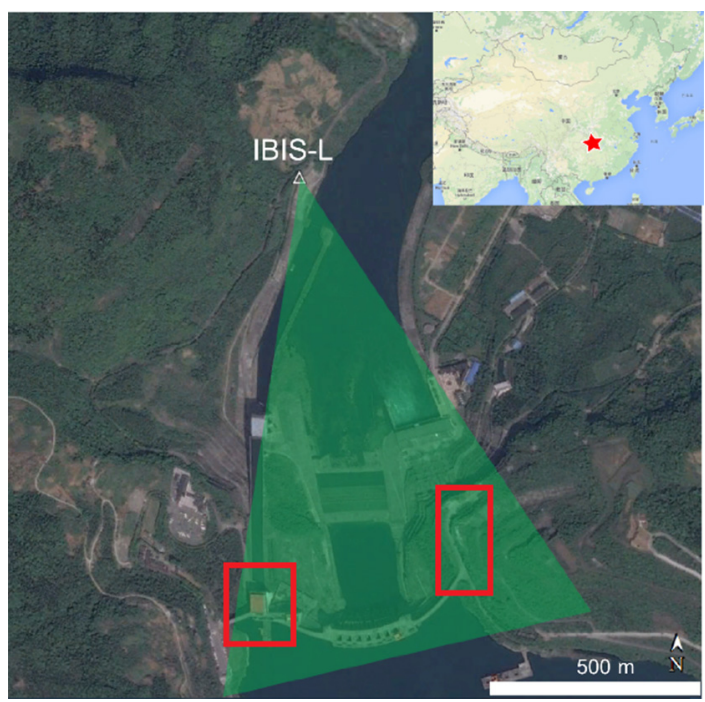

Fig. 2 Geographic location of Geheyan Dam and GB-SAR imaging field of view (green area), the red rectangles marked the area for APS removal. 
Table 1 Main parameters of IBIS-L during the Geheyan campaign.

\begin{tabular}{lc}
\hline \hline & GB-SAR parameters \\
\hline Central frequency/wavelength & $17.1 \mathrm{GHz} / 1.75 \mathrm{~cm}$ \\
Length of the synthetic aperture & $2 \mathrm{~m}$ \\
Maximum distance from target & $1300 \mathrm{~m}$ \\
Range resolution & $0.5 \mathrm{~m}$ \\
Azimuth resolution & $4.4 \mathrm{mrad}$ \\
Azimuth resolution at $1200 \mathrm{~m}$ & $5.3 \mathrm{~m}$ \\
\hline \hline
\end{tabular}
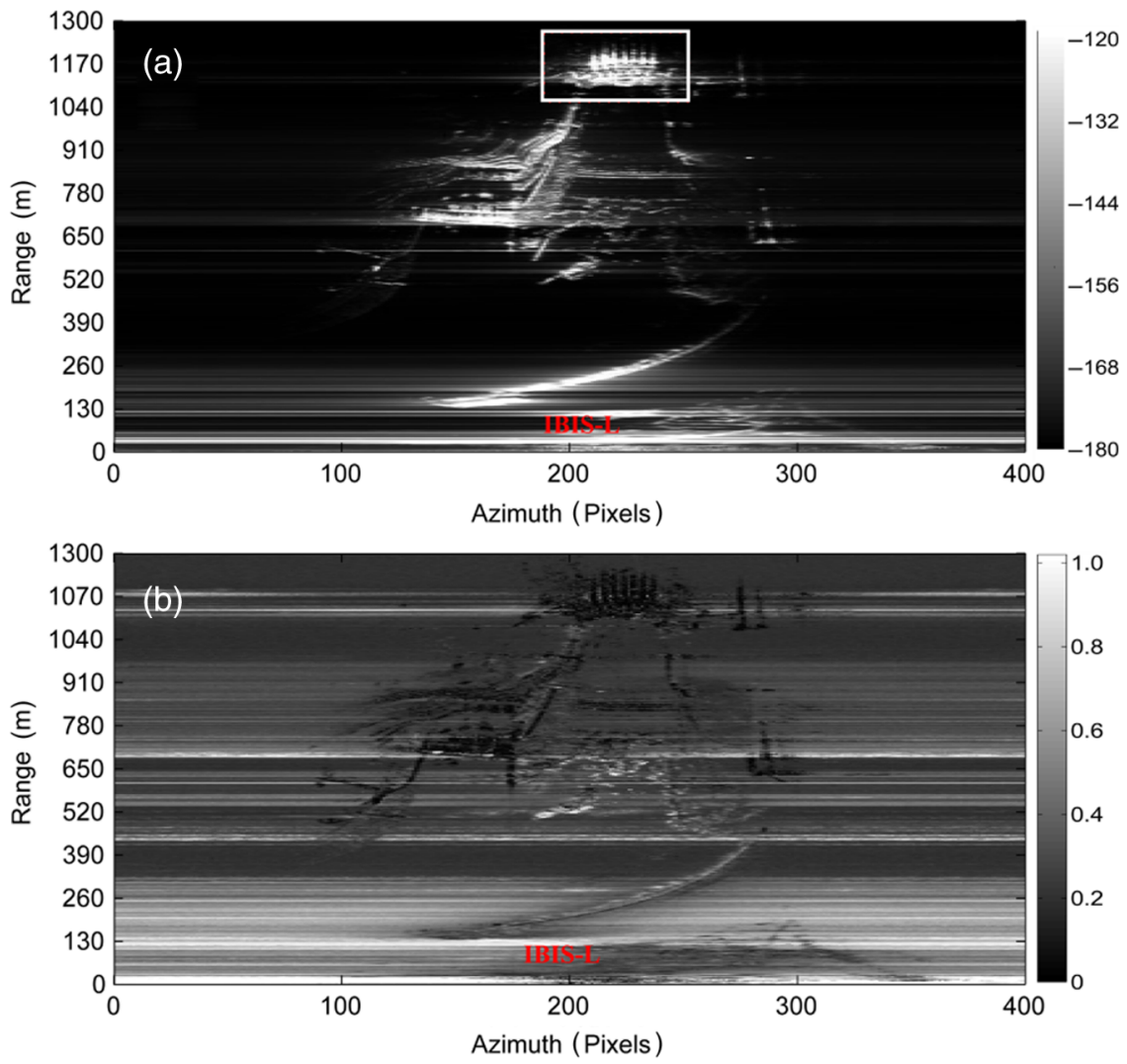

Fig. 3 (a) Mean amplitude in dB with the dam area highlighted by a white rectangle and (b) amplitude dispersion index. Both images are in radar geometry and were calculated using 444 images.

$$
D_{A}=\frac{\sigma_{A}}{m_{A}}
$$

where $m_{A}$ and $\sigma_{A}$ are the mean and standard deviation of the amplitude, respectively. $D_{A}$ of 0.2 was chosen as the threshold. Figure 3(b) shows the amplitude dispersion index where the darker areas are those with lower phase noise.

\section{Data Processing Chain and Strategy}

The flowchart of the procedure used to estimate deformation is shown in Fig. 4. Each step of the procedure is detailed here: 


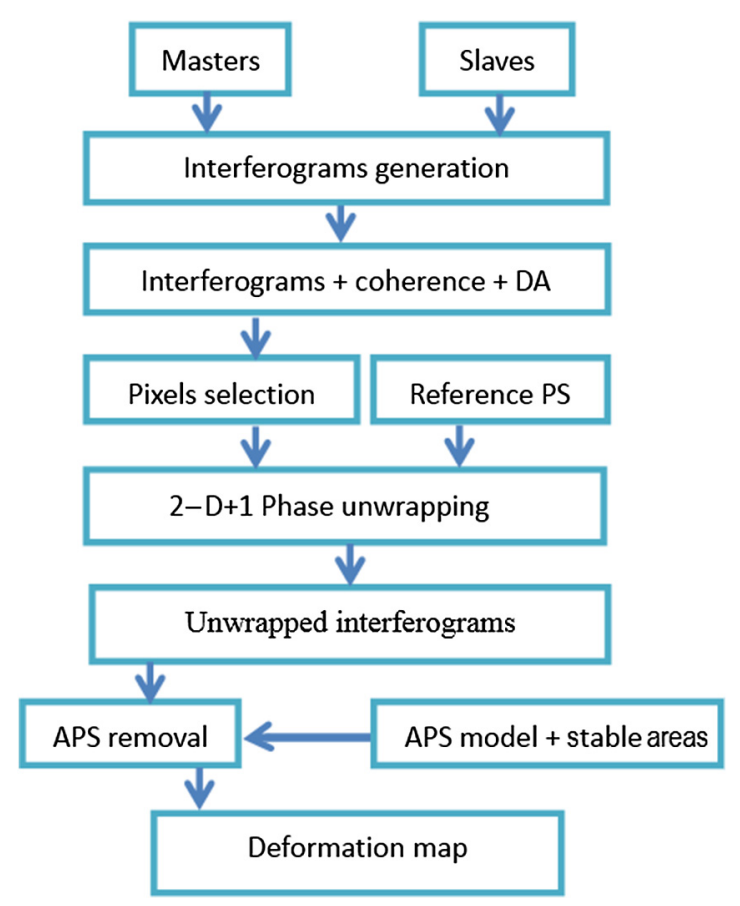

Fig. 4 Flowchart used to estimate deformation on the Geheyan dam campaign.

- GB-SAR image selection: Only 444 images out of 1330 were selected for processing aiming at reducing the computational burden of the huge amount of continuous GB-SAR images acquired. The temporal baseline among images used for analysis is $\sim 16 \mathrm{~min}$.

- Interferogram generation: Interferogram formation is obtained according to the following sequence: for the first selected image, the combinations 1-2, 1-3, 1-4, and 1-5 are calculated, for the second selected image, the combinations are 2-3, 2-4, 2-5, 2-6, and so on. In this way, a total of 1766 interferograms is generated. This approach allows checking the quality of the observations.

- Selection of permanent scatters (PS): ${ }^{16}$ The spatial coherence between every two consecutive images was first estimated, followed by the calculation of the mean coherence. A threshold of 0.4 was then applied to filter out low coherent areas, such as water and vegetated areas. Finally, a threshold of the amplitude dispersion index lower than 0.2 was implemented as a refinement step. The proposed PS selection strategy greatly reduces the calculation effort and decreases the probability of selecting noisy points located close to high coherent points.

- Phase unwrapping: The " $2+1-\mathrm{D}$ " phase unwrapping method developed by the Centre Tecnològic de Telecomunicacions de Catalunya (CTTC) in collaboration with the Department of Earth Sciences of the University of Milan was used. ${ }^{18}$ The two main steps of the method are: (a) a 2-D spatial phase unwrapping is performed on each interferogram generated and (b) a temporal (1-D) phase unwrapping is carried out on each pixel selected. An advantage of this unwrapping strategy is the ability to detect and correct errors that may exist in the 2-D spatial phase unwrapping stage, reducing the error propagation in subsequent processing steps.

- APS removal: As the APS effects are assumed to be strongly spatially correlated in our procedure, a 2-D quadratic polynomial model presented in Ref. 19 is used for APS estimation. On the basis of the plummets behavior, the points on both sides of the dam are first selected as stable areas to estimate the model parameters (see the two red rectangles marked in Fig. 2). Considering the heavy water vapor affecting those points close to the riverbed, they were not used for APS model estimation. Second, the APS was estimated over selected stable areas by least square adjustment performing an outlier rejection. Finally, the estimated model was used over the unstable area to obtain the APS phase component, which was subsequently subtracted from the original phase to obtain an 
APS cleaned phase for the entire set of selected points. The APS removal in terrestrial radar acquisition has been discussed in several papers. ${ }^{20-22}$

\section{Results}

\subsection{GB-SAR Image Interpretation}

Several points were selected to interpret the radar image prior to analyzing and studying the temporal behavior of the dam deformation. Figure 5(a) shows the mean amplitude image calculated using 444 images while a view of the dam from Google Earth ${ }^{\circledR}$ is shown in Fig. 5(b). A comparison of both images allows delineating the crest of the dam (marked with a red dashed line). The best way to interpret a GB-SAR image is to obtain the digital surface model (DSM) of
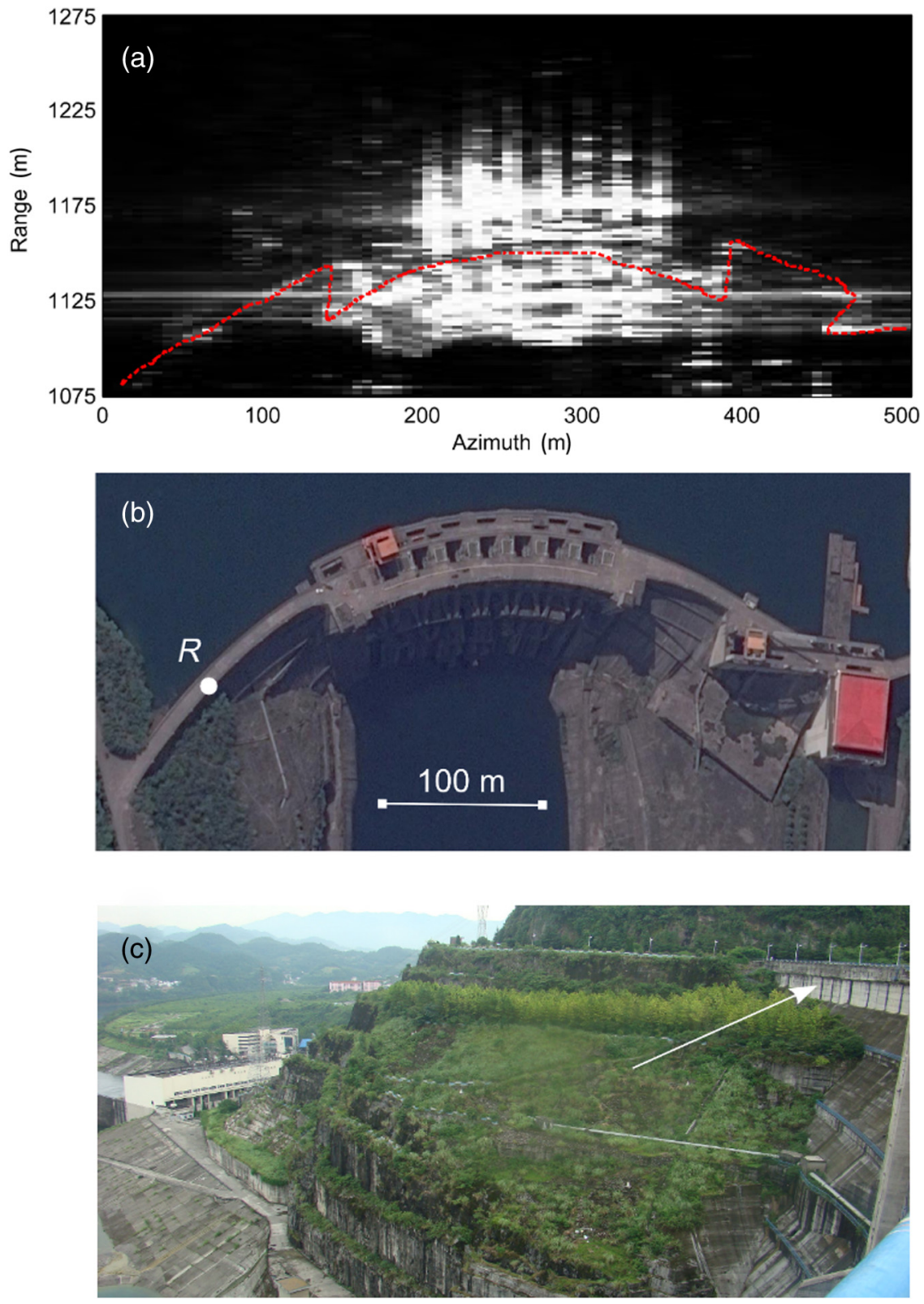

Fig. 5 (a) Zoom of the mean amplitude image obtained from GB-SAR data in radar coordinates. The $Y$-axis indicates the distance in meters from the GB-SAR. The red dashed line highlights the crest of the dam. (b) View of Geheyan Dam from Google Earth ${ }^{\circledR}$. (c) Picture of the dam with the white arrow indicating the location of the reference point $R$. 
the measured area and to geocode the GB-SAR image using this DSM. In this work, a DSM of the dam was not available to assist the interpretation of the GB-SAR image.

As mentioned above, also a GB-SAR can be arranged for DSM generation, but at long distances $(>1000 \mathrm{~m})$, the presence of a high APS can compromise the required accuracy. ${ }^{10}$

The vertical profile of the dam, as shown in Fig. 6, was then analyzed to better understand the radar response of the other parts of the dam in the GB-SAR images. The numbers next to the triangles indicate the height in meters from the bottom of the dam. The GB-SAR was located at $145 \mathrm{~m}$ above sea level, $1145 \mathrm{~m}$ from the dam's middle crest, and it illuminated the entire dam. The elevation of the radar antennas was almost horizontal, with a maximum elevation angle of $\sim 3 \mathrm{deg}$ for the points located at the top of the dam. Considering the thickness of the dam and its downstream face, the pixels closer to the GB-SAR sensor correspond to the lower part of the dam face.

Another important aspect to take into account is the presence of some orifices in the dam. In fact, the radar signal can enter the dam structure through these orifices and suffer multiple reflections in the tunnel (see the red dashed arrows in Fig. 7). An interpretation of the GB-SAR amplitude map shown in Fig. 5(a) can be proposed considering the role of the multipath fading, based on the following issues: (1) the seven dam orifices agree with the number of distinct "tails" visible in this figure, (2) the orifice width agrees with the pixels response in the amplitude image. The azimuth resolution, at a sensor to target distance of $1200 \mathrm{~m}$, is $\sim 5.3 \mathrm{~m}$. Therefore, a 2-3 pixel size in azimuth agrees with the size of the orifices, whose width is $12 \mathrm{~m}$. (3) The depth of the orifices, calculated from the GB-SAR image, is above $80 \mathrm{~m}$, which exceeds the thickness of the dam $(41.2 \mathrm{~m}=12.5+28.7 \mathrm{~m}$, see Fig. 6). An interpretation of this result is that the radar signal is reflected in the hole and, consequently, the sensor to target distance is longer.

The occurrence of unexpected phase delay due to propagation and focusing artifacts (side lobe effect) has been already assessed in Ref. 23. The radar response of highly reflective metallic surfaces (orifice in this case), wet surfaces, can electromagnetically dominate less reflective parts (concrete of the dam structure) and also generate side lobes, as visible in the amplitude images [Figs. 3(a) and 5(a)].

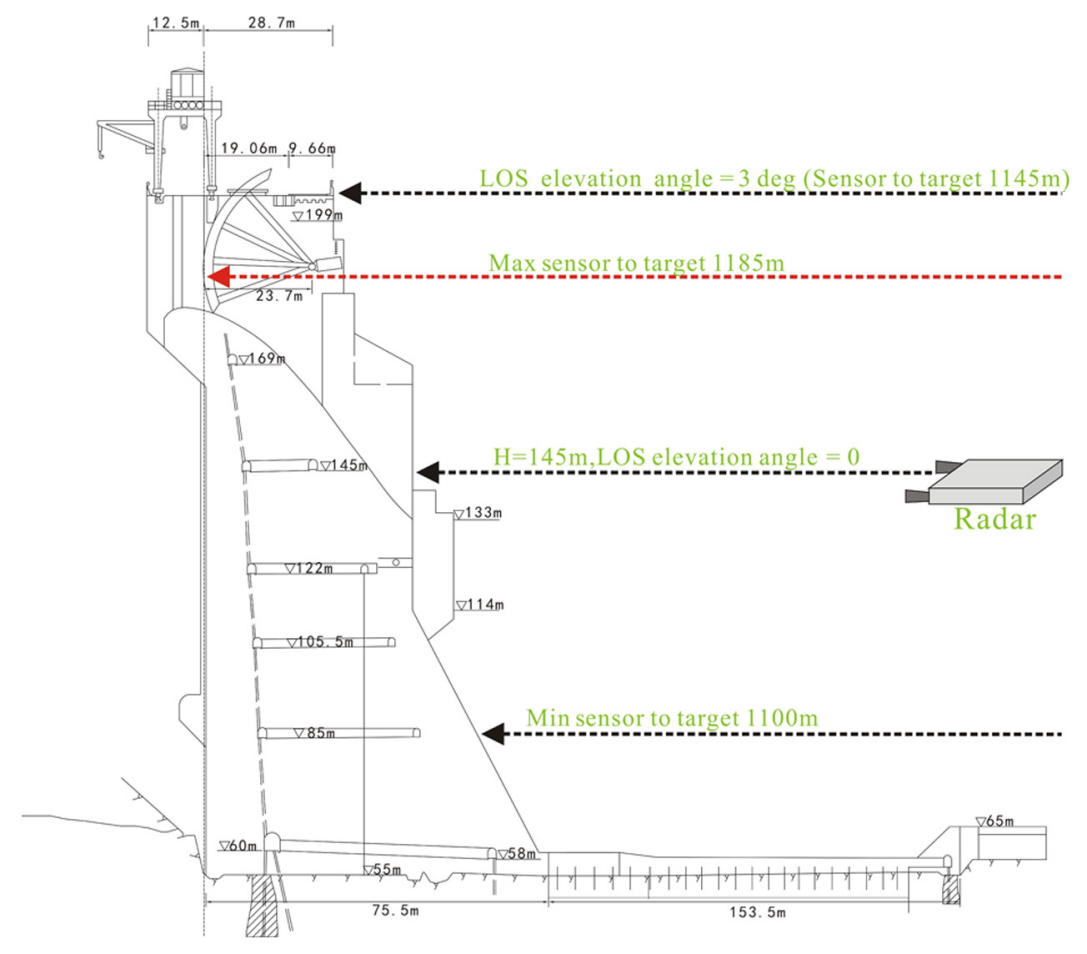

Fig. 6 Profile of the Geheyan Dam indicating the vertical position of the different radar LOS. 


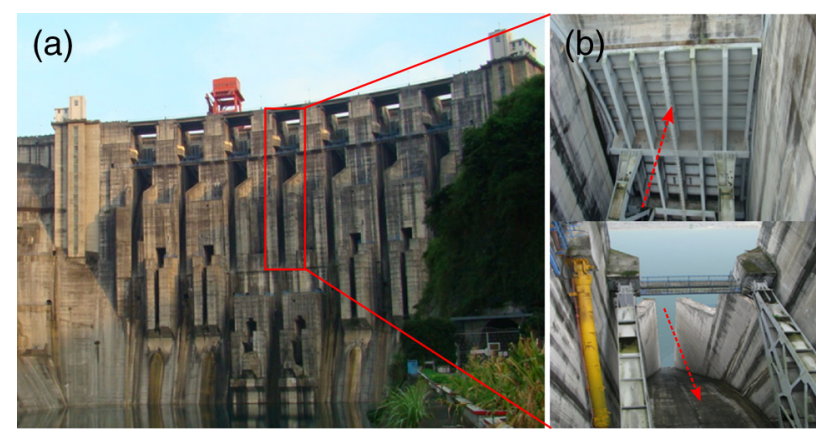

Fig. 7 (a) The dam front face and (b) details of one of the orifices. The red dashed arrows illustrate the main structures affecting the trajectory of the radar signal.

\subsection{Deformation Map and Time Series}

Considering the dam's plummet measurements, we choose the pixel $R$ as the stable, reference point. Figure 8 shows the mean deformation velocity of all the selected points, and the map can be divided into two main sections using the crest of the dam (marked with a dashed red line) as a boundary: a blue section above and a green section below. The blue color indicates that these points are moving toward the radar, while the green color indicates an almost negligible deformation, i.e., no movement. A light blue strip, where a small deformation occurs toward the GB-SAR, can be clearly identified along the crest of the dam.

Figure 9 shows the time series and the calculated linear regression for points D02, D03, and D04 (Fig. 8) selected as representatives of the right, center, and left part of the crest of the dam, respectively. Despite the low correlation coefficients, which are a result of the presence of periodic fluctuations as detailed in Sec. 5, the deformation velocities can be estimated: $-0.17,-0.21$, and $-0.09 \mathrm{~mm} / \mathrm{d}$, for D02, D03, and D04, respectively, as shown in Fig. 10 where the daily average deformation measured in these points is shown. Negative values refer to a reduction of the distance, i.e., motion toward the GB-SAR. The deformation behavior on the crest demonstrates that the central part of the dam shows the highest deformation velocity, which decreases progressively toward the sides.

The dam deformation was also measured using plummets during the GB-SAR campaign. Figure 11 shows the position of plummet PL15, installed in the center of the dam, with the heights of the measurement points marked with red numbers. Figure 12 shows the plummet PL15 measured movements (at different heights) toward the GB-SAR location and the reservoir water level variation during the monitoring campaign. The results indicate that the higher the position with respect to the bottom of the dam, the larger the measured deformation. The vertical behavior of the deformation measured by plummet PL15 at different heights is consistent with GB-SAR

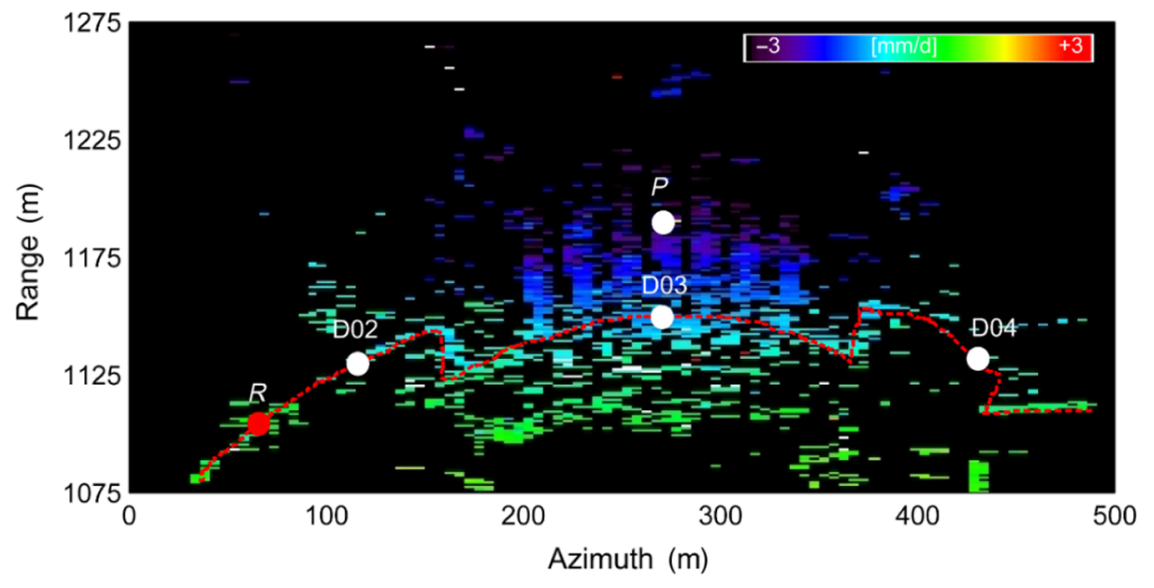

Fig. 8 Mean deformation velocity map. D02, D03, and D04 are points selected on the crest of the dam. $R$ is the stable reference point. $P$ is a point located in one of the orifices of the dam. 

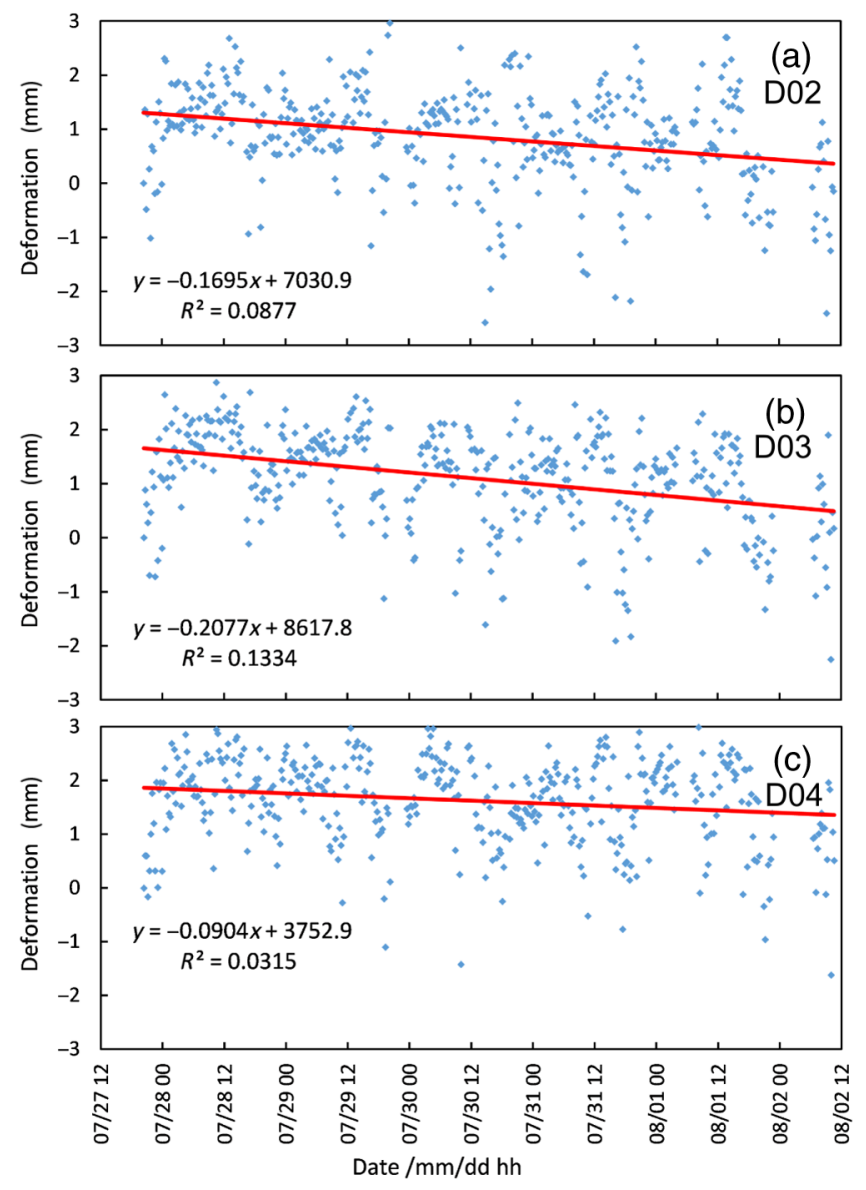

Fig. 9 Deformation time series of the points shown in Fig. 8. The red line indicates the calculated regression line. (a) Point D02, (b) point D03, and (C) point D04.

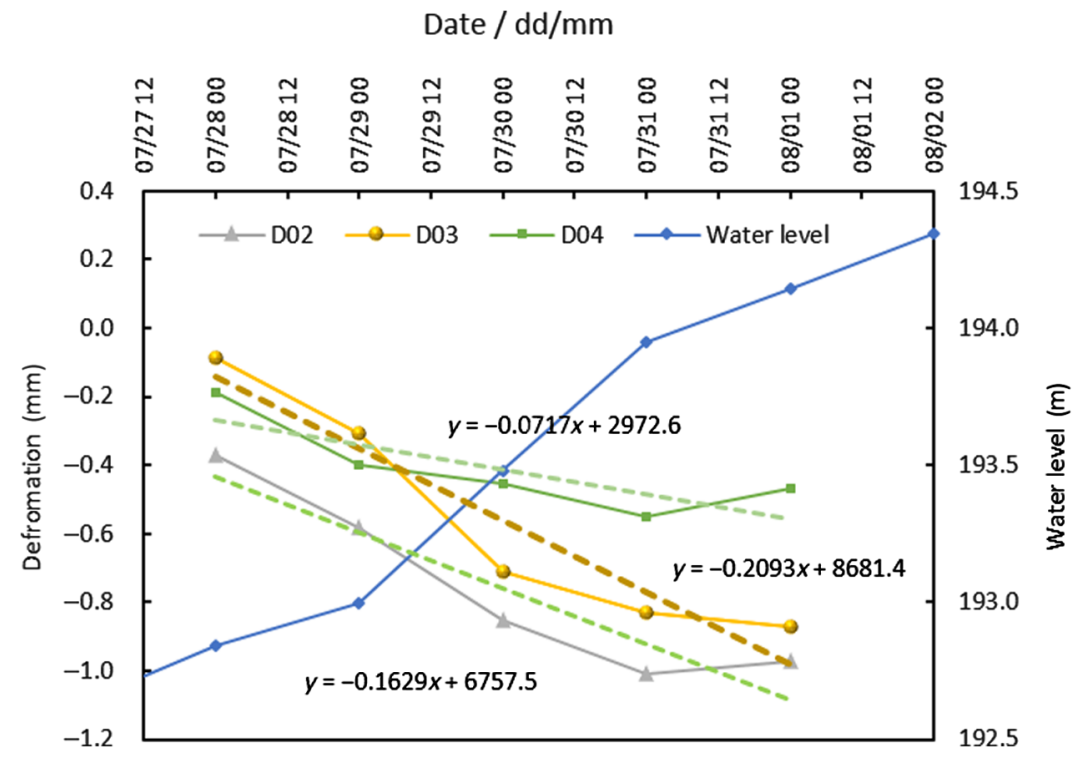

Fig. 10 Daily average deformation measured for points D02, D03, and D04 using the GB-SAR data. The dashed lines indicate the corresponding regression line. The reservoir water level is shown with a solid light blue line. 
(a)

PL15
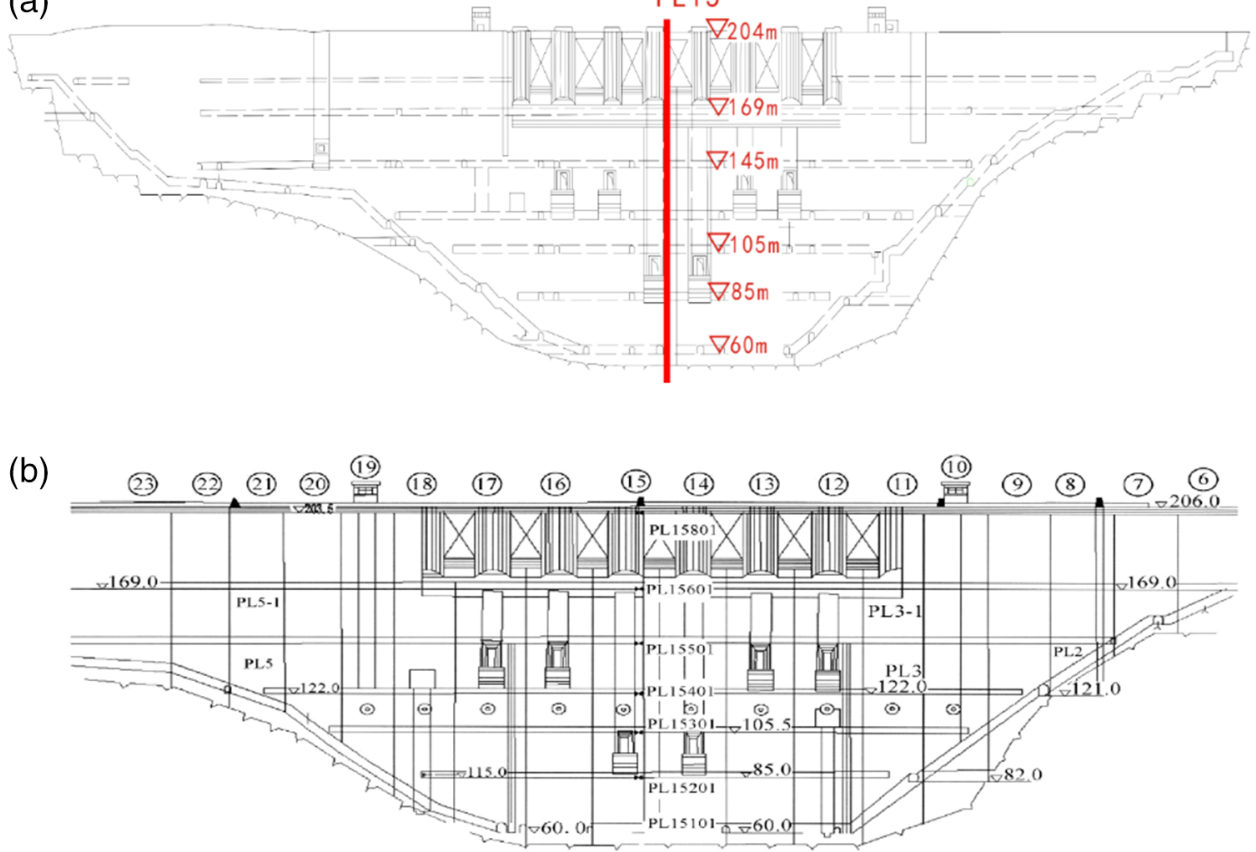

Fig. 11 (a) Vertical profile of the Geheyan Dam indicating the position of plummet PL15, depicted by a red line. The numbers indicate the measurement height. (b) Same view with detailed location of all the installed plummets.

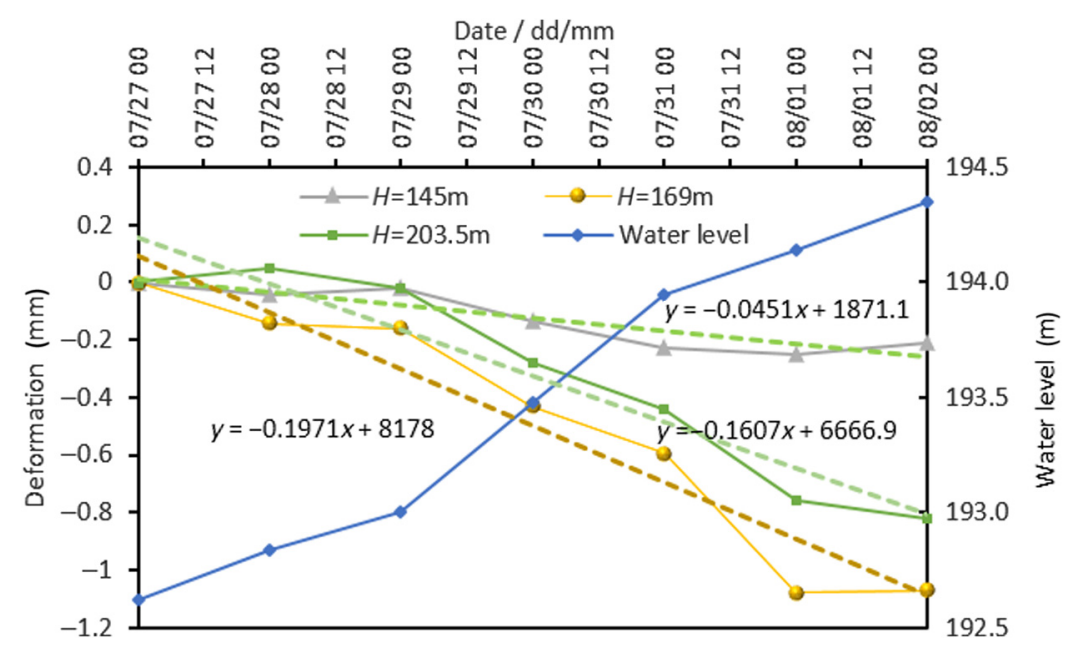

Fig. 12 Deformation showing movement of the dam toward the GB-SAR measured by plummet PL15 at different heights compared with the reservoir water level during the monitoring campaign.

measurements (Table 2, the GB-SAR pixels are selected in the dam center by scaling the height) and the deformation expected when the water reservoir changes. The upper part of the dam shows the highest deformation velocity, which decreases progressively toward the lower part.

It is reasonable that the deformation velocities measured at the dam increase with height. The results shown in Fig. 13 were filtered (moving average with a window size of 9). Time series obtained at the center of the crest of the dam and inside surface orifices are compared, and they indicate that the deformation measured inside and outside the dam at the same height differs. The main interpretation of these results can be summarized as follows. The deformation measured using GB-SAR at the crest of the dam shows good agreement with that measured by plummet PL15 (the purple triangles). Both filtered time series show a cycle of one day, where the 
Table 2 Deformation rate at the center of the dam measured by PL15 and GB-SAR.

\begin{tabular}{lcc}
\hline \hline Point & PL15 $(\mathrm{mm} / \mathrm{d})$ & GB-SAR $(\mathrm{mm} / \mathrm{d})$ \\
\hline PL15 at $H=145 \mathrm{~m}$ & -0.05 & -0.08 \\
PL15 at $H=169 \mathrm{~m}$ & -0.16 & -0.18 \\
PL15 at $H=203 \mathrm{~m}$ & -0.20 & -0.21 \\
\hline \hline
\end{tabular}
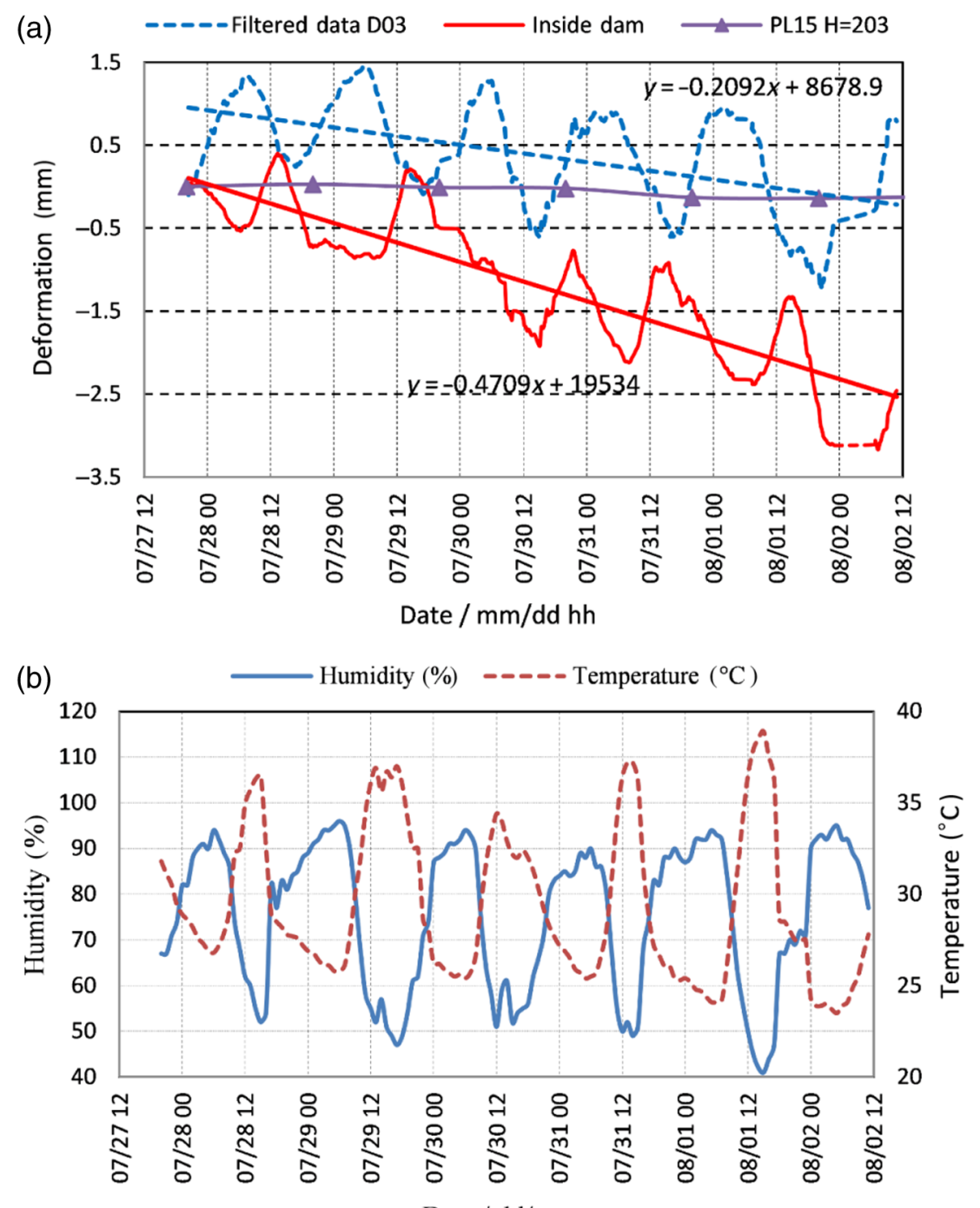

Fig. 13 (a) Deformation time series measured by the GB-SAR at the crest of the dam (D03) and inside one of the orifices (point $P$ shown in Fig. 8 ). The blue dashed line is the deformation time series at point D03, before (oscillating line) and after filtering (straight line); the red line represents the deformation at point $P$, before (oscillating line) and after filtering (straight line); the purple line with triangles refers to the deformation measured by PL15 (at height $203 \mathrm{~m}$ ). (b) Local air temperature (red dashed line) and relative humidity (blue) measured during the GB-SAR campaign.

amplitude ranges between 1 and $1.5 \mathrm{~mm}$. The deformation velocity in the orifice $(-0.47 \mathrm{~mm} / \mathrm{d})$ displays the same trend but it is more than twice that at the crest $(-0.21 \mathrm{~mm} / \mathrm{d})$. Reasonable explanations for these results might be the following: (1) the temporal trend of deformation shows a one day periodic variation which demonstrates there is still residual deformation associated with environmental elements, residual APS, and the different thermal expansions of the 
reference point and of the measured points between the reference point and the measured points are good explanation of the cyclic changes. However, with several days of continuous observations, the estimate of the velocity could be improved, (2) different amplitudes and opposite peaks and troughs may be caused by different atmospheric patterns inside and outside the dam. As stated in Ref. 14, at Ku band, $1 \%$ change of humidity can result in 2-mm radar LOS change in a target-sensor distance of $1000 \mathrm{~m}$, hence, $20 \%$ humidity difference inside the orifice $(40 \mathrm{~m})$ causes 1.6-mm LOS distance change. In high humidity areas, especially in a mountainous area, it is difficult to completely remove the difference of APS inside and outside the structure of the dam, and (3) GB-SAR multireflections are present in the orifices, making the apparent distance sensor to target longer. The reflecting signals are also probably affected by the metal gates, which are influenced by water pressure. Consequently, the gates have a larger magnitude of deformation, as they are not only affected by the dam deformation but also by the distortion caused by the water pressure on the gates. For this reason, a correct interpretation of the GB-SAR data requires an accurate understanding of the amplitude image, a more challenging issue when measurements are taken from long distances, with low azimuth resolution and substantial atmospheric effects.

\section{Conclusions}

The results of a GB-SAR monitoring campaign carried out from more than $1.2 \mathrm{~km}$ far from and aimed at monitoring a large and geometrically complex dam, Geheyan, China, is discussed in this paper. More than 400 GB-SAR images acquired during $\sim 5$ days have been used for interferogram generation. Complex radar signals reflected from the dam have been carefully analyzed by considering the geometry of the sensor and the targets. A 2-D polynomial model has been used for APS estimation and correction. Mean deformation velocities at the main section of the dam have been estimated and compared with plummet measurements. The results show that the maximum deformation velocity estimated by SAR interferometry at the center of the crest of the dam is around $-0.2 \mathrm{~mm} / \mathrm{d}$, in agreement with the measurements of plummets mounted in the dam. Meanwhile, the dam deformation time series are correlated with the reservoir water level during the monitoring campaign. The APS correction applied to interferometric data was mostly satisfactory, except in areas where the atmospheric humidity is very high, and the residual APS clearly shows a one-day periodicity, which does not jeopardize the estimation of deformation velocity over the entire duration of the campaign. The case at hand shows that, due to the large distance radar-dam and the size of the monitored structure, a careful interpretation of the SAR amplitude image is required to associate the deformation behavior of the backscattering inside the discontinuities present in the dam (i.e., the orifices) to avoid an overestimation of the deformation rate. These scatters display similar deformation characteristics as that of the dam front profile, but the deformation velocity can be twice that at the crest of the dam. This difference can be related to the radar signal propagation at the orifices, where the deformation of the metal gates is not only affected by the dam structure but also by the direct pressure of the water contained in the reservoir and the complex variation of the atmospheric parameters. The need to carefully analyze the occurrence of spurious phase shifts in the presence of metal structure and the sidelobes role in radar backscattering in complex GB-SAR scenario seems to agree with assessments of a recent paper. ${ }^{23}$

\section{Acknowledgments}

This work was partly supported by the National Natural Science Foundation of China (No. 41304025). The authors are grateful to Wuhan University for providing the systems used for data acquisition. We acknowledge Gong Jian-bing of Hubei Qingjiang Hydroelectric Development Co., Ltd. for providing support during the GB-SAR campaign. The authors declare no conflict of interest.

\section{References}

1. R. Caduff et al., "A review of terrestrial radar interferometry for measuring surface change in the geosciences," Earth Surf. Processes Landforms 40(2), 208-228 (2015). 
2. O. Monserrat, M. Crosetto, and G. Luzi, "A review of ground-based SAR interferometry for deformation measurement," ISPRS J. Photogramm. Remote Sens. 93, 40-48 (2014).

3. G. Luzi, "Ground based SAR interferometry: a novel tool for geoscience," in Geoscience and Remote Sensing New Achievements, P. Imperatore and D. Riccio, Eds., pp. 1-26, In-Tech, Vukopvar, Croatia (2010).

4. E. Intrieri et al., "Flank instability of Stromboli volcano (Aeolian Islands, Southern Italy): integration of GB-InSAR and geomorphological observations," Geomorphology 201, 60-69 (2013).

5. G. Barla et al., "Monitoring of the Beauregard landslide (Aosta Valley, Italy) using advanced and conventional techniques," Eng. Geol. 116(3-4), 218-235 (2010).

6. D. Tarchi et al., "Landslide monitoring by using ground-based SAR interferometry: an example of application to the Tessina landslide in Italy," Eng. Geol. 68(1-2), 15-30 (2003).

7. K. E. Allstadt et al., "Observations of seasonal and diurnal glacier velocities at Mount Rainier, Washington, using terrestrial radar interferometry," Cryosphere 9(6), 2219-2235 (2015).

8. P. Farina et al., "IBIS-M, an innovative radar for monitoring slopes in open-pit mines," in Proc. of Int. Symp. on Rock Slope Stability in Open Pit Mining and Civil Engineering (2011).

9. G. Nico et al., "Ground-based SAR interferometry for terrain mapping: theory and sensitivity analysis," IEEE Trans. Geosci. Remote Sens. 42(6), 1344-1350 (2004).

10. L. Noferini et al., "DEM by ground-based SAR interferometry," IEEE Geosci. Remote Sens. Lett. 4(4), 659-663 (2007).

11. D. Tarchi et al., "SAR interferometry for structural changes detection: a demonstration test on a dam," in Proc. IEEE Int. Geoscience and Remote Sensing Symp. (IGARSS '99), pp. 1522-1524 (1999).

12. M. Alba et al., "Measurement of dam deformations by terrestrial interferometric techniques," in Int. Archives of the Photogrammetry, Remote Sensing and Spatial Information Sciences, Vol. 37, No. B1, pp. 133-139 (2008).

13. C. Xing, J. J. Huang, and X. Q. Han, "Research on the environmental effects of GB-SAR for dam monitoring," Adv. Mater. Res. 919-921, 392-397 (2014).

14. R. Delsperger et al., "Monitoring of displacements with ground-based microwave interferometry: IBIS-S and IBIS-L," J. Appl. Geod. 4, 41-54 (2010).

15. G. Luzi et al., "Ground-based radar interferometry for landslides monitoring: atmospheric and instrumental decorrelation sources on experimental data," IEEE Trans. Geosci. Remote Sens. 42(11), 2454-2466 (2004).

16. R. F. Hanssen, "Radar Interferometry: Data Interpretation and Error Analysis, p. 308, Kluver Academic Publisher (2001).

17. A. Ferretti, C. Prati, and F. Rocca, "Permanent scatterers in SAR interferometry," IEEE Trans. Geosci. Remote Sens. 39(1), 8-20 (2001).

18. M. Costantini, "A novel phase unwrapping method based on network programming," IEEE Trans. Geosci. Remote Sens. 36(3), 813-821 (1998).

19. N. Devanthéry et al., "An approach to persistent scatterer interferometry," Remote Sens. 6(7), 6662-6679 (2014).

20. L. Noferini et al., "Permanent scatterers analysis for atmospheric correction in ground-based SAR interferometry," IEEE Trans. Geosci. Remote Sens. 43(7), 1459-1471 (2005).

21. R. Iglesias et al., "Atmospheric phase screen compensation in ground-based SAR with a multiple-regression model over mountainous regions," IEEE Trans. Geosci. Remote Sens. 52(5), 2436-2449 (2014).

22. J. Butt, A. Wieser, and S. Conzett, "Intrinsic random functions for mitigation of atmospheric effects in ground based radar interferometry," in Proc. of JISDM, Vienna (2016).

23. M. Frukacz and A. Wieser, "On the impact of rockfall catch fences on ground-based radar interferometry," Landslides 14(4), 1-10 (2017).

Qihuan Huang received his $\mathrm{PhD}$ in geodesy and survey engineering from Hohai University, China, in 2009 and his master's of science from Wuhan University in 2003. He is an associate professor at the School of Earth Sciences and Engineering, Hohai University, China. His 
expertise is in the field of remote sensing for deformation monitoring. He has over 10 years of experience in synthetic aperture radar (SAR) interferometry.

Guido Luzi graduated in physics and holds a $\mathrm{PhD}$ in electronic systems engineering. He has been working since 1986 in microwave remote sensing, passive and active, dedicated to the development of microwave sensors and applications. He published more than 50 papers in refereed international journals. He acts as referee for different journals. He has been with the Geomatics Division of the Centre Tecnològic de Telecomunicacions de Catalunya (CTTC) since January 2014, where his research activities concern the application of spaceborne and terrestrial radar techniques.

Oriol Monserrat received his degree in mathematics from the University of Barcelona and his $\mathrm{PhD}$ in aerospatial science and technology from the Polytechnic University of Catalonia in 2004 and 2012, respectively. Since 2003, he has been working as a researcher, focusing his interest on the processing of remote sensing data from SAR and laser. He participated in different international research projects. He published many papers in international journals. Since January 2015, he has been the head of the Remote Sensing Department, CTTC.

Michele Crosetto holds a civil engineering degree from the Politecnico di Torino in 1993 and a doctorate in geodesy from the Politecnico di Milano in 1998. He has formed part of the Institute of Geomatics since 2002. Since January 2014, he is with CTTC, where he is now head of the Geomatics Division. His main research activity is related to the analysis of spaceborne, airborne and ground-based remote sensing data, and the development of scientific and technical applications. 